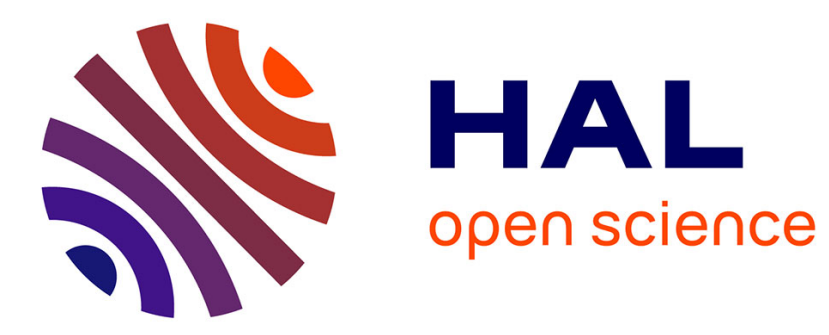

\title{
Regulation of $\sigma$ factors by conserved partner switches controlled by divergent signalling systems
}

\author{
Sophie Bouillet, Dallel Arabet, Cecile Jourlin-Castelli, Vincent Méjean, \\ Chantal Iobbi-Nivol
}

\section{- To cite this version:}

Sophie Bouillet, Dallel Arabet, Cecile Jourlin-Castelli, Vincent Méjean, Chantal Iobbi-Nivol. Regulation of $\sigma$ factors by conserved partner switches controlled by divergent signalling systems. Environmental Microbiology Reports, 2018, 10 (2), pp.127 - 139. 10.1111/1758-2229.12620 . hal-01890037

\section{HAL Id: hal-01890037 \\ https://hal-amu.archives-ouvertes.fr/hal-01890037}

Submitted on 20 Nov 2019

HAL is a multi-disciplinary open access archive for the deposit and dissemination of scientific research documents, whether they are published or not. The documents may come from teaching and research institutions in France or abroad, or from public or private research centers.
L'archive ouverte pluridisciplinaire HAL, est destinée au dépôt et à la diffusion de documents scientifiques de niveau recherche, publiés ou non, émanant des établissements d'enseignement et de recherche français ou étrangers, des laboratoires publics ou privés. 


\section{Regulation of $\sigma$ factors by conserved partner switches controlled by divergent signaling} systems

\section{Sophie Bouillet*, Dallel Arabet, Cécile Jourlin-Castelli, Vincent Méjean and Chantal Iobbi-} Nivol

From Aix-Marseille Univ, CNRS, BIP UMR7281, 13402 Marseille, France.

Running title: Many sensing roads lead to partner switches.

*To whom correspondence should be addressed: Sophie Bouillet, BIP-IMM, CNRS, 31, Chemin Joseph Aiguier, 13402 Marseille Cedex 20, France, E-mail: bouillet.sophie@outlook.fr

Keywords: Environmental signal and stress responses, gene regulation, anti-sigma factor, chemosensory systems, phylogeny.

\section{Abstract}

Partner-Switching Systems (PSS) are widespread regulatory systems, each comprising a kinaseanti- $\sigma$, a phosphorylatable anti- $\sigma$ antagonist and a phosphatase modules. The anti- $\sigma$ domain quickly sequesters or delivers the target $\sigma$ factor according to the phosphorylation state of the anti- $\sigma$ antagonist induced by environmental signals. The PSS components are proteins alone or merged to other domains probably to adapt to the input signals. PSS are involved in major cellular processes including stress response, sporulation, biofilm formation and pathogenesis. Surprisingly, the target $\sigma$ factors are often unknown and the sensing modules acting upstream from the PSS diverge according to the bacterial species. Indeed, they belong to either twocomponent systems or complex pathways as the stressosome or Chemosensory Systems (CS). 
Based on a phylogenetic analysis, we propose that the sensing module in Gram-negative bacteria is often a CS.

\section{Introduction}

Bacteria have to face constantly many environmental changes in their ecological niches. Their ability to rewire rapidly the expression of genes involved in the response to these alterations is crucial for their survival and is mediated by the use of different alternative $\sigma$ factors. The $\sigma$ factors target the core RNA polymerase to the promoter of genes required to adapt to new cell surroundings. All bacteria possess a primary housekeeping $\sigma$ factor $\left(\sigma^{70}\right)$ that insures the transcription of the majority of genes, but most bacteria own additional $\sigma$ factors that coordinate the transcription of many genes involved in response and adaptation of bacteria to changing environmental or cellular conditions. The number of these alternative $\sigma$ factors varies depending on the lifestyle of the bacteria (Österberg et al., 2011).

Except for Escherichia coli $\sigma^{54}$ and homologs, all $\sigma$ factors belong to the $\sigma^{70}$ family, divided itself into four subfamilies based on phylogenetic and structural properties (Paget and Helmann, 2003; Helmann, 2011). The group 1 of $\sigma^{70}$ family is composed of the whole housekeeping $\sigma$ factors, $\sigma^{70}$ also called $\sigma^{\mathrm{A}}$ in Gram-positive bacteria. The group 2 gathers $\sigma$ factors that resemble to that of group 1 except that they are not essential in laboratory conditions, as $\sigma^{\text {s }}$ factor involved in the General Stress Response (GSR) of proteobacteria (Helmann, 2011; Battesti et al., 2011). The group 3 contains more diverse $\sigma$ factors, which have roles in various cellular processes as $\sigma^{32}$ that regulates genes involved in response to heat shock, $\sigma^{28}$ in flagella synthesis and also $\sigma^{\mathrm{E}}, \sigma^{\mathrm{F}}, \sigma^{\mathrm{G}}, \sigma^{\mathrm{K}}$, of Bacillus subtilis that control different stages of sporulation. The group 4 contains very diverse $\sigma$ factors called $\sigma^{\mathrm{ECF}}$ (for ExtraCytoplasmic Function) that have been classified into at least 56 subgroups (Staroń et al., 2009; Huang et al., 2015; Sineva et al., 2017).

Sigma factors play crucial roles to conduct the cellular processes and in turn they have to be tightly regulated. In many cases, sigma factors are controlled at several levels, from transcription to post-translation. At the protein level, we can distinguish three main modes of regulation, the proteolysis of the sigma factor as for $\sigma^{S}$ in E. coli (Becker et al., 1999; Hengge, 2009, 2011; 
Battesti et al., 2011); the cleavage of the $\sigma$ factor from an inactive to an active form, including pro- $\sigma^{\mathrm{E}}$ and pro- $\sigma^{\mathrm{K}}$ in B. subtilis (Hilbert and Piggot, 2004; Higgins and Dworkin, 2012; Fimlaid and Shen, 2015) and the inactivation of the sigma factor activity by an anti- $\sigma$ factor (Hughes and Mathee, 1998; Helmann, 1999; Österberg et al., 2011; Feklístov et al., 2014; Paget, 2015). The anti- $\sigma$ factors sequester their target $\sigma$ factor, disabling it to interact with the core RNAP and thus to transcribe genes of its regulon (Campbell et al., 2002; Sorenson et al., 2004; Feklístov et al., 2014). In doing so, anti- $\sigma$ factors could protect their $\sigma$ factor partner from proteolysis (Barembruch and Hengge, 2007; Mao et al., 2013, 2014; Bouillet et al., 2017). Thus, anti- $\sigma$ factors enable the cell to maintain a pool of $\sigma$ factor molecules that can be rapidly released to act without de novo synthesis when suddenly required. Most of the $\sigma^{\mathrm{ECF}}$ are regulated by anti- $\sigma$ factors and are usually co-transcribed into the same transcriptional unit to keep a 1:1 stoichiometry of the two proteins (Brooks and Buchanan, 2008; Campagne et al., 2015). The release of the $\sigma$ factor is also precisely driven to ensure the transcription of its regulon in response to specific signals. Different strategies are thus employed including cell-surface signaling (regulation of $\sigma^{24}$ also called $\sigma^{\mathrm{E}}$ in E. coli) (Brooks and Buchanan, 2008; Ho and Ellermeier, 2012), the secretion of the anti- $\sigma$ factors (FliA/FlgM in E. coli) (Hughes et al., 1993; Kutsukake, 1994; Smith and Hoover, 2009), the direct sensing of redox state by cysteine residues of the anti- $\sigma$ factors (RsrA/ $\sigma^{\mathrm{R}}$ in Streptomyces coelicolor) (Ilbert et al., 2006; Jung et al., 2011) as well as the involvement of an additional protein called anti- $\sigma$ factor antagonist in a mechanism known as the partner-switching system (PSS).

\section{Partner-switching systems}

The term "partner-switch" has been defined by Alper and colleagues in 1994 to describe the mechanism that regulates $\sigma^{\mathrm{F}}$, crucial in the sporulation process of B. subtilis (Alper et al., 1994).

The nature of the PSS components as well as their function is a common feature among typical PSS. Indeed, PSS are made up with an anti- $\sigma$ factor having a serine kinase activity (HATPase domain), a phosphorylatable STAS anti- $\sigma$ antagonist, a PP2C serine phosphatase and a target $\sigma$ factor (Alper et al., 1994; Mittenhuber, 2002). Anti- $\sigma$ factors are constituted of a dimerization 
interface and of a HATPase domain. The latter harbors conserved motifs for phosphorylation and is found in many kinase families (Dutta and Inouye, 2000). STAS stands for Sulfate Transporter and Anti-Sigma antagonist and proteins containing this domain play a role in either of these two processes and are phosphorylatable on a specific serine residue (Sharma et al., 2011). The PP2C domain characterizes a family of serine/threonine phosphatases that need metallic ion for their activity (Shi, 2009; Pereira et al., 2011; Bradshaw et al., 2017).

In a partner-switching mechanism, the anti- $\sigma$ factor binds to its target $\sigma$ factor disabling it to recruit the core RNAP. The release of the $\sigma$ factor is mediated by the anti- $\sigma$ factor antagonist, also called anti-anti- $\sigma$ factor. Indeed, when no specific signal is transduced to the regulatory system, the anti- $\sigma$ factor binds to the $\sigma$ factor and phosphorylates its partner the anti- $\sigma$ factor antagonist. When the specific signal arises, the PP2C-type phosphatase is activated and dephosphorylates the anti- $\sigma$ factor antagonist that becomes thus efficient to interact with the anti- $\sigma$, leading to the release of the $\sigma$ factor by a competition effect. Anti- $\sigma$ factors have usually a better affinity for the unphosphorylated form of the anti-anti- $\sigma$ factor than for the $\sigma$ protein allowing the binding of the $\sigma$ factor only when the anti- $\sigma$ factor antagonist is phosphorylated but not when it is dephosphorylated (Duncan et al., 1996; Masuda et al., 2004; Bouillet et al., 2016). A 3D-structure of the anti- $\sigma$ factor SpoIIAB bound to its partners showed that two monomers of anti- $\sigma$ factors interact with only one monomer of $\sigma^{\mathrm{F}}$ factor but with two monomers of anti- $\sigma$ factor antagonist SpoIIAA (Campbell et al., 2002; Masuda et al., 2004). This mode of binding is probably similar for other PSS. Moreover, anti- $\sigma$ proteins contain a dimerization interface suggesting their ability to dimerize. However, additional biochemical characterization of the interactions of the PSS partners is needed to confirm their mode of action.

Numerous PSS have been discovered in many bacteria including Gram-negative bacteria whereas they were initially thought to be restricted to Gram-positive bacteria (Shi et al., 1999; Mittenhuber, 2002; Kozak et al., 2005; Morris and Visick, 2010; Houot et al., 2012; Morris and Visick, 2013b; Eshghi et al., 2014; Mercer and Lang, 2014; Lambert et al., 2015; Thompson et al., 2015; Bouillet et al., 2016; Gebhardt and Shuman, 2017). Although PSS comprises four 
components (an anti- $\sigma$ factor, an anti- $\sigma$ factor antagonist, a phosphatase and a $\sigma$ factor), the domain organization of the partners is highly diversified as depicted in Figure 1. While the anti- $\sigma$ antagonist usually remains as a one-domain protein, the anti- $\sigma$ factor can be a domain of a complex protein. Indeed, it can be associated with other domains including receiver domain of typical response regulator, PP2C-type phosphatase domain or unknown function domain. Interestingly, the phosphatase is usually associated with a signaling domain such as receiver, HAMP or detection domains. These data show that PSS have evolved probably according to the detected stresses and to the target $\sigma$ factor.

In conclusion, although the core domain composition is conserved, the domain organization frequently varies and, consequently, PSS often comprise additional domains (Figure 1) (Mittenhuber, 2002; Galperin, 2006).

Nevertheless, the presence of PSS encoded within bacterial genomes remains yet poorly studied. Phylogenetic analyses of PSS are thus needed to evaluate how widespread are these systems in particular in Gram-negative bacteria. In fact, the presence of HATPase and STAS domains in other proteins and complex domain organizations make difficult the characterization of anti- $\sigma$ and anti-anti- $\sigma$ factors in bacteria by using basic bioinformatics.

However, anti- $\sigma$ factor antagonist and phosphatase homologs have been found in Gram-positive bacteria, as well as cyanobacteria, Deinococcus species and proteobacteria including Pseudomonas, Vibrio and Shewanella species (Mittenhuber, 2002). For example, B. subtilis and B. cereus possess $16 \sigma$ factors and two known PSS targeting $\sigma^{\mathrm{F}}$ and $\sigma^{\mathrm{B}}$, M. tuberculosis harbors $13 \sigma$ factors, one of which is subjected to two PSS $\left(\sigma^{\mathrm{F}}\right.$ is submitted to UsfX/RsfAB and to Rv1364c regulation). In $S$. oneidensis, $10 \sigma$ factors are present with a known and a putative PSS. Surprisingly, some species including E. coli do not seem to possess PSS. In contrast, the Grampositive bacterium $S$. coelicolor is one of the organisms that contain the highest number of $\sigma$ factors (60 to 65) and its chromosome has been predicted to encode many PSS partners with 45 anti- $\sigma$ factors, 18 anti-anti- $\sigma$ factors and 44 PP2C proteins (Bentley et al., 2002; Mittenhuber, 2002; Martínez et al., 2009). Among them, few have been identified but their study appears 
complicated because of cross-talks between several PSS, and anti- $\sigma$ factor antagonists could have more than one associated anti- $\sigma$ factor. If we consider that each anti- $\sigma$ factor antagonist is the output protein from distinct sensory modules, this suggests that the release and thus the activation of the targeted $\sigma$ factor might be induced by many transducing pathways in response to various signals.

\section{Role of the Partner-switching systems}

The role of PSS is to control the availability of specific $\sigma$ factors. However, various PSS have been discovered in many bacteria but the targeted $\sigma$ factor has not been found in some cases (Figure 1).

PSS signal transduction pathways seem to be implicated in various major cellular processes including the control of type III secretion system synthesis, virulence, chemotaxis, biofilm formation, exoprotein production, stress responses and also metabolism (Shi et al., 1999; Mattoo et al., 2004; Kozak et al., 2005; Bordi et al., 2010; Bhuwan et al., 2012; Houot et al., 2012; Morris and Visick, 2013b, 2013a; Eshghi et al., 2014; Mercer and Lang, 2014; Lambert et al., 2015; Bouillet et al., 2016; Gebhardt and Shuman, 2017). For instance, the spore formation of B. subtilis is a complex multi-step mechanism under the control of many regulators (Higgins and Dworkin, 2012; Fimlaid and Shen, 2015). Notably, four main $\sigma$ factors act successively during the sporulation process. Each of them is thus tightly regulated but has to be also quickly freed to make sure that all genes are correctly expressed in time. $\sigma^{\mathrm{F}}$ is active during the first stage of sporulation only in the forespore compartment. The anti- $\sigma$ factor SpoIIAB interacts with $\sigma^{\mathrm{F}}$ disabling it to recruit the core RNAP. The release of $\sigma^{\mathrm{F}}$ is permitted by the anti- $\sigma$ factor antagonist SpoIIAA. When no signal is transduced to the regulatory system, SpoIIAB binds to $\sigma^{\mathrm{F}}$ and also phosphorylates SpoIIAA. When a signal is launched, the PP2C-type phosphatase SpoIIE, which is a membrane-anchored protein that perceives the signal dephosphorylates specifically SpoIIAA. The anti- $\sigma$ antagonist becomes thus efficient to interact with SpoIIAB, leading to the release of $\sigma^{\mathrm{F}}$ (Diederich et al., 1994; Duncan et al., 1996; Magnin et al., 1997; Campbell et al., 2002; Masuda et al., 2004; Levdikov et al., 2012). 
The Rsb partner-switching system regulating the availability of the $\sigma^{\mathrm{B}}$ factor of Bacillales is another PSS that has been extensively studied. $\sigma^{\mathrm{B}}$, the general stress response (GSR) $\sigma$ factor, is inhibited during growth conditions without stress by the anti- $\sigma$ factor RsbW and $\sigma^{\mathrm{B}}$ becomes active during stress conditions due to the binding of the dephosphorylated anti- $\sigma$ factor antagonist RsbV on RsbW (Figures 1 and 2) (Price, 2011).

Beside Gram-positive bacteria PSS models, it has been recently shown in Shewanella oneidensis, an aquatic bacterium from the $\gamma$-proteobacteria class, $\sigma^{S}$, the GSR- $\sigma$ factor, is post-translationally regulated by a PSS (Figures 1 and 2E). This system is composed of the two main proteins CrsA and CrsR, an anti- $\sigma$ factor antagonist and a three-domain response regulator, respectively. CrsR contains a N-terminal receiver, a central PP2C and a C-terminal HATPase domains. The latter is able to phosphorylate efficiently CrsA whereas the phosphatase domain dephosphorylates it. In addition, direct interactions of CrsR-CrsA and of $\mathrm{CrsR}-\sigma^{\mathrm{S}}$ combined with in vivo data have revealed that $\sigma^{S}$ is the target of the PSS. The CrsRA- $\sigma^{S}$ is the first system that links GSR regulation to PSS in a $\gamma$-proteobacterium as it is the case in many other bacterial species (Bouillet et al., 2016, 2017).

In P. aeruginosa, the PSS protein HsbR comprises a receiver, a PP2C phosphatase and a HATPase domains. It has been proposed that the last domain could constitute a simple kinase and not an anti- $\sigma$ factor (Figure 1). The anti- $\sigma$ factor antagonist HsbA can bind to the anti- $\sigma$ factor FlgM, inducing the release of the $\sigma$ factor FliA (or $\sigma^{28}$ ) (Bhuwan et al., 2012). Furthermore, HsbA is subjected to phosphorylation control by HsbR that, consequently, modulates the activity of HsbA. Contrarily to typical PSS, HsbA acts thus downstream HsbR. This system controls swimming motility through the regulation of the flagella synthesis mediated by FliA. Furthermore, HsbA interacts with the diguanylate cyclase HsbD stimulating therefore the c-diGMP production (Valentini et al., 2016). This original partner-switch has thus crucial functional implications in both motility control and biofilm development.

The Syp system formed by SypE and SypA of Vibrio fisheri as well as the Btr system composed of BtrW and BtrV of Bordetella pertussis have important roles in biofilm formation and 
pathogenesis through type III secretion system control, respectively (Figure 1). The target $\sigma$ factor and the mechanism of action have not been unraveled. Interestingly, as for HsbR of $P$. aeruginosa, the HATPase domains of SypE and RsbW could act solely as a kinase and not as an anti- $\sigma$ factor (Kozak et al., 2005; Morris and Visick, 2013b, 2013a).

Usually, PSS regulate the activity of alternative $\sigma$ factors but one example of a housekeeping sigma factor regulation by a PSS has been recently brought to light (Figure 1). Indeed, the primary $\sigma$ factor $\sigma^{66}$ of Chlamydia trachomatis is controlled by PSS partners (Hua et al., 2006; Thompson et al., 2015).

\section{Atypical partner-switching modules}

In E. coli, $\sigma^{70}$ activity is also modulated by the anti- $\sigma$ factor Rsd and the histidine phosphorylatable HPr that acts as an anti- $\sigma$ factor antagonist. However, even though the mechanism of sequestration and release of the $\sigma$ factor is alike that of PSS, the two partners Rsd and HPr are not homologous to typical PSS proteins with HPr playing a primary role in the translocation of several sugars across the membrane (Mitchell et al., 2007; Yuan et al., 2008; Hofmann et al., 2011; Park et al., 2013, 2015).

In Gram-negative $\alpha$-proteobacterial species, the $\sigma$ factor controlling the GSR called $\sigma^{\mathrm{ECFG}}$, RpoE or SigT depending on the bacterium, is controlled by an atypical PSS (Figure 2F) (Francez-Charlot et al., 2009; Staroń et al., 2009; Campagne et al., 2012; Kaczmarczyk et al., 2014; Kim et al., 2014; Francez-Charlot et al., 2015; Fiebig et al., 2015; Herrou et al., 2015; Francez-Charlot et al., 2016). Indeed, the PSS partners are not homologous to canonical PSS proteins. Nevertheless, they use a similar mechanism of sequestration and release of the $\sigma$ factor as typical PSS. The PSS module of GSR regulation is mostly shared by $\alpha$-proteobacterial species with little divergences. In all cases, two proteins are involved: the anti- $\sigma$ factor NepR and the two-domain PhyR. The latter acts as an anti- $\sigma$ factor antagonist and contains a $\mathrm{N}$-terminal $\sigma$-like factor and a C-terminal receiver domains (Figure 2F). NepR possesses homologies with $\sigma^{\mathrm{ECF}}$ factors. Phosphorylation of the receiver domain increases the affinity between the $\sigma$-like domain of PhyR and NepR so that the $\sigma^{\text {ECF }}$ is freed whereas unphosphorylated PhyR has almost no affinity for NepR that in turn binds 
to $\sigma^{\mathrm{ECFG}}$. The phosphorylation state of PhyR is controlled by various histidine kinases that detect and transduce signals including blue light and osmolytes. As for GSR regulation of Gram-positive bacteria, the composition and the number of the sensory inputs vary greatly from a bacterium to another depending on their lifestyle (Fiebig et al., 2015; Francez-Charlot et al., 2015).

\section{Activation of partner-switching systems}

Diversified sensory and transducing modules appear to converge to PSS, although the mechanisms of transduction have not been unraveled in most cases. Input modules could be two-component systems, chemosensory systems or might be directly integrated in PSS modules via the addition of sensing domain(s) in PSS proteins (Figures 1 and 2) (Hsu et al., 2008; Morris and Visick, 2013a; Lambert et al., 2015; Norsworthy and Visick, 2015; Chambonnier et al., 2016). In the Gram-positive bacteria Bacillus, Listeria and Staphyloccoccus, the stress responsive $\sigma$ factor $\sigma^{\mathrm{B}}$ is submitted to a PSS. The PSS module formed by the anti- $\sigma$ factor RsbW and the anti- $\sigma$ factor antagonist RsbV is conserved but the RsbV phosphatases as well as the mechanisms of signal transduction to the PSS module diverge from one species to another (Figure 2A and 2B).

In B. subtilis, two sensing modules linked to two independent PP2C-containing phosphatases (RsbU and RsbP) converge to the PSS module. Environmental stresses including blue light, heat shock and osmolytes are detected by a protein complex called the stressosome that subsequently transduces signals to the PP2C phosphatase RsbU. The energetic level of the cell is perceived by the PAS domain containing phosphatase RsbP associated with the hydrolase RsbQ (Figure 2A) (Benson and Haldenwang, 1993; Boylan et al., 1993; Voelker et al., 1996; Kim et al., 2004; Marles-Wright et al., 2008; Marles-Wright and Lewis, 2010; Price, 2011; Gaidenko and Price, 2014; Guldimann et al., 2016). RsbU, RsbP and a stressosome are absent from other Bacillales as B. cereus. They are replaced by the RsbY protein composed of a receiver fused to a PP2C domain and the complex histidine kinase RsbK, which is able to detect internal and environmental stresses and thus transduces the signal to RsbY (Figure 2B) (van Schaik et al., 2005; de Been et al., 2010, 2011). Interestingly, genes coding for a stressosome-like complex are also found in many species in particular among the proteobacteria, cyanobacteria and 
actinobacteria phyla (Pané-Farré et al., 2005; Jia et al., 2016). Likewise, homologs of the histidine kinase RsbK have been found in many species including the proteobacterial genus Vibrio, Pseudomonas, Magnetoccocus or Myхococcus but their roles are still unknown (de Been et al., 2011).

Streptomyces coelicolor PSS regulation of $\sigma^{\mathrm{B}}$ resembles that of $B$. subtilis but possesses an additional PSS absent from other species (Lee et al., 2004): the Osa system that regulates $\sigma^{\mathrm{B}}$ under "back to normal" conditions after an osmotic shock. OsaA is a RsbK homolog that may detect signals from a GAF domain, $\mathrm{OsaB}$ is a two-domain protein with a $\mathrm{N}$-terminal receiver and an unknown C-terminal domain, and OsaC contains an anti- $\sigma$ factor, a PAS, two GAF and a PP2Ctype phosphatase domains (Figure 2C) (Martínez et al., 2009; Price, 2011). The regulatory cascade that regulates $\sigma^{\mathrm{B}}$ availability is still unknown but the domain composition of OsaC suggests a direct additional signal sensing by the phosphatase protein. In the Actinomycetales Mycobacterium tuberculosis, the GSR $\sigma$ factor called $\sigma^{\mathrm{F}}$ is also mediated by two PSS (DeMaio et al., 1997). Notably, the protein Rv1364c is a PSS module organized in four domains corresponding to a PAS, a phosphatase, an anti- $\sigma$ factor and an anti- $\sigma$ factor antagonist domain (Parida et al., 2005; Sachdeva et al., 2008; Greenstein et al., 2009; Malik et al., 2009; Jaiswal et al., 2010; KingScott et al., 2011). It has been shown that its anti- $\sigma$ factor domain can bind to $\sigma^{\mathrm{F}}$ whereas its anti$\sigma$ factor antagonist domain antagonizes the action of the anti- $\sigma$ domain. However, the complex network of $\sigma^{\mathrm{F}}$ post-translational regulation has not been completely unraveled yet, but Rv1364c seems to detect signals itself without upstream sensing module.

The PSS composed of HsbR and HsbA from P. aeruginosa is activated by a complex phosphocascade. The histidine phosphotransfer protein HptB constitutes the module activating HsbR. HptB acts in the GacA-GacS two-component pathway regulating sRNA involved in $P$. aeruginosa biofilm formation and pathogenesis (Lin et al., 2006; Hsu et al., 2008; Bordi et al., 2010; Bhuwan et al., 2012; Houot et al., 2012; Chambonnier et al., 2016). Moreover, other histidine kinases have been shown to detect specific signals and transduce them by 
phosphorylating HptB that in turn phosphorylates the receiver domain of $\mathrm{HsbR}$, activating the phosphatase domain of HsbR (Hsu et al., 2008).

\section{Activation of partner-switching modules in various proteobacteria: a role for} chemosensory systems

Chemosensory systems (CS) are complex signal transduction pathways mainly involve in the regulation of the flagella rotation necessary for swimming motility in most bacteria. Since then, many CS that do not control bacterial motility have been spotted in various bacteria. They were shown to play a role in the cellular differentiation of Myxococcus xanthus and Rhodospirillum centenum, in the production of molecules involved in biofilm formation as c-di-GMP or EPS in $P$. aeruginosa and Azospirillum brasilense or in the type IV pili based motility in P. aeruginosa. In fact, a large number of bacteria possess more than one CS coding locus in their genome, but their roles are still largely unknown (Kirby, 2009; Wuichet and Zhulin, 2010; He and Bauer, 2014).

In S. oneidensis, the genes coding for the two PSS proteins CrsA and CrsR are located in the che1 locus, composed of 10 genes with 8 of them coding for classical CS components (Che proteins). This system is not involved in swimming motility and could form a chemosensory system (Armitano et al., 2013). This gene organization strongly suggests that the chemosensory system Che1 contains the signal sensing machinery that regulates the activity of the CrsR-CrsA PSS. Interestingly, it appears that chemosensory machinery adapted to regulate a partner-switch is a common feature of aquatic proteobacteria (Figure 3). Indeed, crsR gene homologs are widespread among those bacteria and an analysis of the genes surrounding $\operatorname{crs} R$ in 59 bacterial genomes was carried out for this review (Bouillet et al., 2017). This phylogenetic analysis clearly shows that the CrsR-CrsA PSS is most of the time genetically related to a che locus. As shown in Figure 3, the $\operatorname{crs} R$-crsA genes are located in the vicinity of a central histidine kinase cheA gene in $71 \%$ of these bacteria (32 out of 45), or of two-component histidine kinase(s). This indicates that the sensing modules of the CrsR-CrsA partner-switch could predominantly be a chemosensory system. Interestingly, two genes coding for detectors MCP (Methyl-accepting Chemotaxis Proteins) are comprised in the che1 locus of S. oneidensis. One of them is predicted 
to be anchored to the membrane whereas the other seems to be located in the cytosol and possesses two PAS domains (Figure 2). The two MCP could thus allow the detection of different kinds of signals: one from outside and the other from inside the cell cytoplasm. Interestingly, many PSS controlling the GSR including those in B. cereus and in some alpha-proteobacterial species often comprise two sensory detectors presenting similar sensing domains as those found in MCP (Figure 2 and Figure 3). It has been shown that the RsbK histidine kinase from B. cereus that controls the downstream RsbVWY PSS is subjected to the methylation by the methyltransferase RsbM, as usually seen in MCP (Chen et al., 2012, 2015). The methylation of RsbK by RsbM leads to the inhibition of $\sigma^{\mathrm{B}}$. As the che1 locus of $S$. oneidensis contains a gene encoding the methyltransferase CheR1, we propose that $\sigma^{\mathrm{S}}$ sequestration by CrsR could also be modulated by the methylation level of the two MCP.

PSS imbedded in a CS operon has been recently described in Leptospira interrogans. This operon contains genes encoding a response regulator composed of a receiver and an anti- $\sigma$ factor domains, an anti- $\sigma$ factor antagonist and chemosensory proteins (CheA, CheY, CheW, CheD, CheB, MCP). This PSS that controls a still unknown $\sigma$ factor could be regulated by the chemosensory system (Eshghi et al., 2014; Lambert et al., 2015).

Two-component systems or signal transduction coding genes have also been found in the neighborhood of $\operatorname{crs} R$ homologs and could thus be the sensor that detects signals and transduces them to the PSS. Genes encoding Hpt proteins are also found near to crsR-crsA genes in Hahella ganghwensis and Marinobacter lipolyticus (Figure 3). This illustrates that the sensing modules acting upstream PSS diverge from one species to another.

\section{The case of GSR regulation in bacteria}

The main strategy commonly developed by bacteria to respond, defend and adapt to general stresses is to modify its transcriptional program in order to express appropriate genes. This ability is mediated by the use of a specific $\sigma$ factor. Gram-positive bacteria as Bacillales and Actinomycetales possess a type-3 $\sigma$ factor named $\sigma^{\mathrm{B}}$ or $\sigma^{\mathrm{F}}$, whereas $\beta$ - and $\gamma$-proteobacteria hold the type- $2 \sigma$ factor $\sigma^{S}$ and $\alpha$-proteobacteria use a type- $4 \sigma^{\mathrm{ECF}}$ often called $\sigma^{\mathrm{ECFG}}$ (Boylan et al., 
1993; Battesti et al., 2011; Hengge, 2011; Price, 2011; Fiebig et al., 2015; Francez-Charlot et al., 2015). Despite the fact that all these $\sigma$ factors are not homologous and present large differences in terms of sequence and structure, they control analogous processes in the cells (AlvarezMartinez et al., 2006; Sauviac et al., 2007; Gourion et al., 2009; Martínez-Salazar et al., 2009; Britos et al., 2011; Hengge, 2011; Foreman et al., 2012; Jans et al., 2013; Kim et al., 2013; Landini et al., 2014; Guldimann et al., 2016). Moreover, although their global regulation is highly divergent, the presence of a PSS is a relatively common feature for their post-translational regulation (Figure 2).

Strikingly, although B. cereus and S. oneidensis are distant species and use the two nonhomologous GSR $\sigma$ factors $\sigma^{\mathrm{B}}$ and $\sigma^{\mathrm{S}}$, respectively, the pathways that control their posttranslational activity are similar. Conversely, E. coli and S. oneidensis are both $\gamma$-proteobacteria and both possess $\sigma^{\mathrm{S}}$ to regulate GSR, but their post-translational regulations of $\sigma^{\mathrm{S}}$ are entirely different (Battesti et al., 2011; Price, 2011). This strongly suggests that these regulatory pathways can be more related between bacteria that share common lifestyle than phylogenetic proximity.

\section{Concluding Remarks}

Since a couple of years, novel PSS have been detected in many bacteria. These PSS play a key role in major cellular processes although the partner $\sigma$ factor has not been determined in several cases. PSS are common post-translational regulators for the control of the response to general stress but they are also involved in crucial cell processes as motility, biofilm formation, virulence, and cell differentiation as sporulation. Other physiological roles of PSS will be undoubtedly discovered in the next future.

In fact, based solely on basic bioinformatics, it is quite difficult to find out PSS genes within bacterial genomes mainly because their HATPase domain is common to other types of proteins. Furthermore, each species has to adapt to its own environment, doing this, it has set up dedicated regulatory pathways. PSS and the sensing modules have thus evolved to adapt to their target $\sigma$ factors and to the signals this $\sigma$ factor has to be responding to. As a consequence, the 
domain organization of the PSS (Figures 1 and 2) and the composition of the sensing modules vary greatly (Figures 2 and 3). In other words, a common signal transduction pathway like PSS can be activated by a large range of sensing machineries.

In conclusion, recent results have revealed that PSS are found not only in Gram-positive but also in Gram-negative bacteria. Moreover, many PSS are governed by complex signaling pathways including two-component and chemosensory systems. PSS are clearly very efficient and rapid ways to trigger or stop specific $\sigma$ factor responses. Therefore, future studies will most probably reveal novel module architectures for PSS and their regulatory pathways to better respond to the numberless environmental signals encountered by bacteria.

\section{Acknowledgements}

We would like to thank the anonymous reviewers for helpful suggestions, members of the group, in particular Olivier Lemaire for fruitful discussions and Olivier Genest for discussions and reviewing the manuscript. We also thank David Redelberger for assistance with drawings. This work was supported by the Centre National de la Recherche Scientifique and Aix-Marseille Université (AMU). S.B. was supported by a Ministère de l'Enseignement Supérieur et de la Recherche fellowship and AMU.

Conflict of interest: The authors declare that they have no conflicts of interest with the contents of this article.

\section{References}

Alper, S., Duncan, L., and Losick, R. (1994) An adenosine nucleotide switch controlling the activity of a cell type-specific transcription factor in B. subtilis. Cell 77: 195-205.

Alvarez-Martinez, C.E., Baldini, R.L., and Gomes, S.L. (2006) A Caulobacter crescentus Extracytoplasmic Function Sigma Factor Mediating the Response to Oxidative Stress in Stationary Phase. J. Bacteriol. 188: 1835-1846.

Armitano, J., Méjean, V., and Jourlin-Castelli, C. (2013) Aerotaxis governs floating biofilm formation in Shewanella oneidensis. Environ. Microbiol. 15: 3108-3118.

Barembruch, C. and Hengge, R. (2007) Cellular levels and activity of the flagellar sigma factor FliA of Escherichia coli are controlled by FlgM-modulated proteolysis. Mol. Microbiol. 65: 76-89.

Battesti, A., Majdalani, N., and Gottesman, S. (2011) The RpoS-mediated general stress response in Escherichia coli. Annu. Rev. Microbiol. 65: 189-213. 
Becker, G., Klauck, E., and Hengge-Aronis, R. (1999) Regulation of RpoS proteolysis in Escherichia coli: The response regulator RssB is a recognition factor that interacts with the turnover element in RpoS. Proc. Natl. Acad. Sci. U. S. A. 96: 6439-6444.

de Been, M., Francke, C., Siezen, R.J., and Abee, T. (2011) Novel sigmaB regulation modules of Gram-positive bacteria involve the use of complex hybrid histidine kinases. Microbiol. Read. Engl. 157: 3-12.

de Been, M., Tempelaars, M.H., van Schaik, W., Moezelaar, R., Siezen, R.J., and Abee, T. (2010) A novel hybrid kinase is essential for regulating the sigma(B)-mediated stress response of Bacillus cereus. Environ. Microbiol. 12: 730-745.

Benson, A.K. and Haldenwang, W.G. (1993) Regulation of sigma B levels and activity in Bacillus subtilis. J. Bacteriol. 175: 2347-2356.

Bentley, S.D., Chater, K.F., Cerdeño-Tárraga, A.-M., Challis, G.L., Thomson, N.R., James, K.D., et al. (2002) Complete genome sequence of the model actinomycete Streptomyces coelicolor A3(2). Nature 417: 141-147.

Bhuwan, M., Lee, H.-J., Peng, H.-L., and Chang, H.-Y. (2012) Histidine-containing phosphotransfer protein-B (HptB) regulates swarming motility through partner-switching system in Pseudomonas aeruginosa PA01 strain. J. Biol. Chem. 287: 1903-1914.

Bordi, C., Lamy, M.-C., Ventre, I., Termine, E., Hachani, A., Fillet, S., et al. (2010) Regulatory RNAs and the HptB/RetS signalling pathways fine-tune Pseudomonas aeruginosa pathogenesis. Mol. Microbiol. 76: 1427-1443.

Bouillet, S., Genest, O., Jourlin-Castelli, C., Fons, M., Méjean, V., and Iobbi-Nivol, C. (2016) The General Stress Response $\sigma$ S Is Regulated by a Partner Switch in the Gram-negative Bacterium Shewanella oneidensis. J. Biol. Chem. 291: 26151-26163.

Bouillet, S., Genest, O., Méjean, V., and Iobbi-Nivol, C. (2017) Protection of the General Stress Response $\sigma(\mathrm{S})$ by CrsR Allows a Rapid and Efficient Adaptation of Shewanella oneidensis. J. Biol. Chem.

Boylan, S.A., Redfield, A.R., Brody, M.S., and Price, C.W. (1993) Stress-induced activation of the sigma B transcription factor of Bacillus subtilis. J. Bacteriol. 175: 7931-7937.

Bradshaw, N., Levdikov, V.M., Zimanyi, C.M., Gaudet, R., Wilkinson, A.J., and Losick, R. (2017) A widespread family of serine/threonine protein phosphatases shares a common regulatory switch with proteasomal proteases. eLife 6:.

Britos, L., Abeliuk, E., Taverner, T., Lipton, M., McAdams, H., and Shapiro, L. (2011) Regulatory response to carbon starvation in Caulobacter crescentus. PloS One 6: e18179.

Brooks, B.E. and Buchanan, S.K. (2008) Signaling mechanisms for activation of extracytoplasmic function (ECF) sigma factors. Biochim. Biophys. Acta 1778: 1930-1945.

Campagne, S., Allain, F.H.-T., and Vorholt, J.A. (2015) Extra Cytoplasmic Function sigma factors, recent structural insights into promoter recognition and regulation. Curr. Opin. Struct. Biol. 30: 71-78.

Campagne, S., Damberger, F.F., Kaczmarczyk, A., Francez-Charlot, A., Allain, F.H.-T., and Vorholt, J.A. (2012) Structural basis for sigma factor mimicry in the general stress response of Alphaproteobacteria. Proc. Natl. Acad. Sci. U. S. A. 109: E1405-1414.

Campbell, E.A., Masuda, S., Sun, J.L., Muzzin, O., Olson, C.A., Wang, S., and Darst, S.A. (2002) Crystal Structure of the Bacillus stearothermophilus Anti- $\sigma$ Factor SpoIIAB with the Sporulation $\sigma$ Factor $\sigma \mathrm{F}$. Cell 108: 795-807.

Chambonnier, G., Roux, L., Redelberger, D., Fadel, F., Filloux, A., Sivaneson, M., et al. (2016) The Hybrid Histidine Kinase LadS Forms a Multicomponent Signal Transduction System with 
the GacS/GacA Two-Component System in Pseudomonas aeruginosa. PLoS Genet. 12: e1006032.

Chen, J.-C., Liu, J.-H., Hsu, D.-W., Shu, J.-C., Chen, C.-Y., and Chen, C.-C. (2015) Methylatable Signaling Helix Coordinated Inhibitory Receiver Domain in Sensor Kinase Modulates Environmental Stress Response in Bacillus Cereus. PLoS ONE 10:.

Chen, L.-C., Chen, J.-C., Shu, J.-C., Chen, C.-Y., Chen, S.-C., Chen, S.-H., et al. (2012) Interplay of RsbM and RsbK controls the $\sigma \mathrm{B}$ activity of Bacillus cereus. Environ. Microbiol. 14: 2788-2799.

DeMaio, J., Zhang, Y., Ko, C., and Bishai, W.R. (1997) Mycobacterium tuberculosis sigF is part of a gene cluster with similarities to the Bacillus subtilis sigF and sigB operons. Tuber. Lung Dis. Off. J. Int. Union Tuberc. Lung Dis. 78: 3-12.

Dereeper, A., Guignon, V., Blanc, G., Audic, S., Buffet, S., Chevenet, F., et al. (2008) Phylogeny.fr: robust phylogenetic analysis for the non-specialist. Nucleic Acids Res. 36: W465-469.

Diederich, B., Wilkinson, J.F., Magnin, T., Najafi, M., Erringston, J., and Yudkin, M.D. (1994) Role of interactions between SpoIIAA and SpoIIAB in regulating cell-specific transcription factor sigma F of Bacillus subtilis. Genes Dev. 8: 2653-2663.

Duncan, L., Alper, S., and Losick, R. (1996) SpoIIAA governs the release of the cell-type specific transcription factor sigma F from its anti-sigma factor SpoIIAB. J. Mol. Biol. 260: 147164.

Dutta, R. and Inouye, M. (2000) GHKL, an emergent ATPase/kinase superfamily. Trends Biochem. Sci. 25: 24-28.

Eshghi, A., Becam, J., Lambert, A., Sismeiro, O., Dillies, M.-A., Jagla, B., et al. (2014) A putative regulatory genetic locus modulates virulence in the pathogen Leptospira interrogans. Infect. Immun. 82: 2542-2552.

Feklístov, A., Sharon, B.D., Darst, S.A., and Gross, C.A. (2014) Bacterial sigma factors: a historical, structural, and genomic perspective. Annu. Rev. Microbiol. 68: 357-376.

Fiebig, A., Herrou, J., Willett, J., and Crosson, S. (2015) General Stress Signaling in the Alphaproteobacteria. Annu. Rev. Genet. 49: null.

Fimlaid, K.A. and Shen, A. (2015) Diverse mechanisms regulate sporulation sigma factor activity in the Firmicutes. Curr. Opin. Microbiol. 24: 88-95.

Foreman, R., Fiebig, A., and Crosson, S. (2012) The LovK-LovR Two-Component System Is a Regulator of the General Stress Pathway in Caulobacter crescentus. J. Bacteriol. 194: 3038-3049.

Francez-Charlot, A., Frunzke, J., Reichen, C., Ebneter, J.Z., Gourion, B., and Vorholt, J.A. (2009) Sigma factor mimicry involved in regulation of general stress response. Proc. Natl. Acad. Sci. U. S. A. 106: 3467-3472.

Francez-Charlot, A., Frunzke, J., Zingg, J., Kaczmarczyk, A., and Vorholt, J.A. (2016) Multiple $\sigma E c f G$ and NepR Proteins Are Involved in the General Stress Response in Methylobacterium extorquens. PLoS ONE 11:.

Francez-Charlot, A., Kaczmarczyk, A., Fischer, H.-M., and Vorholt, J.A. (2015) The general stress response in Alphaproteobacteria. Trends Microbiol. 23: 164-171.

Gaidenko, T.A. and Price, C.W. (2014) Genetic evidence for a phosphorylation-independent signal transduction mechanism within the Bacillus subtilis stressosome. PloS One 9: e90741.

Galperin, M.Y. (2006) Structural Classification of Bacterial Response Regulators: Diversity of Output Domains and Domain Combinations. J. Bacteriol. 188: 4169-4182.

Gebhardt, M.J. and Shuman, H.A. (2017) GigA and GigB are Master Regulators of Antibiotic Resistance, Stress Responses and Virulence in Acinetobacter baumannii. J. Bacteriol. 
Gourion, B., Sulser, S., Frunzke, J., Francez-Charlot, A., Stiefel, P., Pessi, G., et al. (2009) The PhyRsigma(EcfG) signalling cascade is involved in stress response and symbiotic efficiency in Bradyrhizobium japonicum. Mol. Microbiol. 73: 291-305.

Greenstein, A.E., Hammel, M., Cavazos, A., and Alber, T. (2009) Interdomain communication in the Mycobacterium tuberculosis environmental phosphatase Rv1364c. J. Biol. Chem. 284: 29828-29835.

Guldimann, C., Boor, K.J., Wiedmann, M., and Guariglia-Oropeza, V. (2016) Resilience in the face of uncertainty: sigma B fine-tunes gene expression to support homeostasis in Grampositive bacteria. Appl. Environ. Microbiol. AEM.00714-16.

He, K. and Bauer, C.E. (2014) Chemosensory signaling systems that control bacterial survival. Trends Microbiol. 22: 389-398.

Helmann, J.D. (1999) Anti-sigma factors. Curr. Opin. Microbiol. 2: 135-141.

Helmann J.D. (2011) Regulation by Alternative Sigma Factors. In, Bacterial Stress Responses, 2nd ed. Gisela Storz and Regine Hengge, pp. 31-43.

Hengge, R. (2009) Proteolysis of sigmaS (RpoS) and the general stress response in Escherichia coli. Res. Microbiol. 160: 667-676.

Hengge, R. (2011) The General Stress Response in Gram-negative Bacteria. In, Bacterial Stress Responses, 2nd edition, ASM. Storz, Gisela and Hengge, Regine, p. 251.

Herrou, J., Willett, J.W., and Crosson, S. (2015) Structured and Dynamic Disordered Domains Regulate the Activity of a Multifunctional Anti- $\sigma$ Factor. mBio 6: e00910-15.

Higgins, D. and Dworkin, J. (2012) Recent progress in Bacillus subtilis sporulation. FEMS Microbiol. Rev. 36: 131-148.

Hilbert, D.W. and Piggot, P.J. (2004) Compartmentalization of gene expression during Bacillus subtilis spore formation. Microbiol. Mol. Biol. Rev. MMBR 68: 234-262.

Ho, T.D. and Ellermeier, C.D. (2012) Extra cytoplasmic function $\sigma$ factor activation. Curr. Opin. Microbiol. 15: 182-188.

Hofmann, N., Wurm, R., and Wagner, R. (2011) The E. coli anti-sigma factor Rsd: studies on the specificity and regulation of its expression. PloS One 6: e19235.

Houot, L., Fanni, A., de Bentzmann, S., and Bordi, C. (2012) A bacterial two-hybrid genome fragment library for deciphering regulatory networks of the opportunistic pathogen Pseudomonas aeruginosa. Microbiol. 158: 1964-1971.

Hsu, J.-L., Chen, H.-C., Peng, H.-L., and Chang, H.-Y. (2008) Characterization of the histidinecontaining phosphotransfer protein B-mediated multistep phosphorelay system in Pseudomonas aeruginosa PA01. J. Biol. Chem. 283: 9933-9944.

Hua, L., Hefty, P.S., Lee, Y.J., Lee, Y.M., Stephens, R.S., and Price, C.W. (2006) Core of the partner switching signalling mechanism is conserved in the obligate intracellular pathogen Chlamydia trachomatis. Mol. Microbiol. 59: 623-636.

Huang, X., Pinto, D., Fritz, G., and Mascher, T. (2015) Environmental Sensing in Actinobacteria: a Comprehensive Survey on the Signaling Capacity of This Phylum. J. Bacteriol. 197: 25172535.

Hughes, K.T., Gillen, K.L., Semon, M.J., and Karlinsey, J.E. (1993) Sensing structural intermediates in bacterial flagellar assembly by export of a negative regulator. Science 262: 12771280.

Hughes, K.T. and Mathee, K. (1998) The anti-sigma factors. Annu. Rev. Microbiol. 52: 231-286. Ilbert, M., Graf, P.C.F., and Jakob, U. (2006) Zinc center as redox switch--new function for an old motif. Antioxid. Redox Signal. 8: 835-846. 
Jaiswal, R.K., Manjeera, G., and Gopal, B. (2010) Role of a PAS sensor domain in the Mycobacterium tuberculosis transcription regulator Rv1364c. Biochem. Biophys. Res. Commun. 398: 342-349.

Jans, A., Vercruysse, M., Gao, S., Engelen, K., Lambrichts, I., Fauvart, M., and Michiels, J. (2013) Canonical and non-canonical EcfG sigma factors control the general stress response in Rhizobium etli. MicrobiologyOpen 2: 976-987.

Jia, X., Wang, J., Rivera, S., Duong, D., and Weinert, E.E. (2016) An 02-sensing stressosome from a Gram-negative bacterium. Nat. Commun. 7: 12381.

Jung, Y.-G., Cho, Y.-B., Kim, M.-S., Yoo, J.-S., Hong, S.-H., and Roe, J.-H. (2011) Determinants of redox sensitivity in RsrA, a zinc-containing anti-sigma factor for regulating thiol oxidative stress response. Nucleic Acids Res. 39: 7586-7597.

Kaczmarczyk, A., Hochstrasser, R., Vorholt, J.A., and Francez-Charlot, A. (2014) Complex twocomponent signaling regulates the general stress response in Alphaproteobacteria. Proc. Natl. Acad. Sci. U. S. A. 111: E5196-5204.

Kim, H.-S., Caswell, C.C., Foreman, R., Roop, R.M., and Crosson, S. (2013) The Brucella abortus General Stress Response System Regulates Chronic Mammalian Infection and Is Controlled by Phosphorylation and Proteolysis. J. Biol. Chem. 288: 13906-13916.

Kim, H.-S., Willett, J.W., Jain-Gupta, N., Fiebig, A., and Crosson, S. (2014) The Brucella abortus virulence regulator, LovhK, is a sensor kinase in the general stress response signalling pathway. Mol. Microbiol. 94: 913-925.

Kim, T.-J., Gaidenko, T.A., and Price, C.W. (2004) In vivo phosphorylation of partner switching regulators correlates with stress transmission in the environmental signaling pathway of Bacillus subtilis. J. Bacteriol. 186: 6124-6132.

King-Scott, J., Konarev, P.V., Panjikar, S., Jordanova, R., Svergun, D.I., and Tucker, P.A. (2011) Structural characterization of the multidomain regulatory protein Rv1364c from Mycobacterium tuberculosis. Struct. Lond. Engl. 1993 19: 56-69.

Kirby, J.R. (2009) Chemotaxis-like regulatory systems: unique roles in diverse bacteria. Annu. Rev. Microbiol. 63: 45-59.

Kozak, N.A., Mattoo, S., Foreman-Wykert, A.K., Whitelegge, J.P., and Miller, J.F. (2005) Interactions between partner switcher orthologs BtrW and BtrV regulate type III secretion in Bordetella. J. Bacteriol. 187: 5665-5676.

Krummenacker, M., Paley, S., Mueller, L., Yan, T., and Karp, P.D. (2005) Querying and computing with BioCyc databases. Bioinforma. Oxf. Engl. 21: 3454-3455.

Kutsukake, K. (1994) Excretion of the anti-sigma factor through a flagellar substructure couples flagellar gene expression with flagellar assembly in Salmonella typhimurium. Mol. Gen. Genet. MGG 243: 605-612.

Lambert, A., Wong Ng, J., and Picardeau, M. (2015) Gene inactivation of a chemotaxis operon in the pathogen Leptospira interrogans. FEMS Microbiol. Lett. 362: 1-8.

Landini, P., Egli, T., Wolf, J., and Lacour, S. (2014) sigmaS, a major player in the response to environmental stresses in Escherichia coli: role, regulation and mechanisms of promoter recognition. Environ. Microbiol. Rep. 6: 1-13.

Lee, E.-J., Cho, Y.-H., Kim, H.-S., Ahn, B.-E., and Roe, J.-H. (2004) Regulation of $\sigma$ B by an Anti- and an Anti-Anti-Sigma Factor in Streptomyces coelicolor in Response to Osmotic Stress. J. Bacteriol. 186: 8490-8498.

Levdikov, V.M., Blagova, E.V., Rawlings, A.E., Jameson, K., Tunaley, J., Hart, D.J., et al. (2012) Structure of the phosphatase domain of the cell fate determinant SpoIIE from Bacillus subtilis. J. Mol. Biol. 415: 343-358. 
Lin, C.-T., Huang, Y.-J., Chu, P.-H., Hsu, J.-L., Huang, C.-H., and Peng, H.-L. (2006) Identification of an HptB-mediated multi-step phosphorelay in Pseudomonas aeruginosa PA01. Res. Microbiol. 157: 169-175.

Magnin, T., Lord, M., and Yudkin, M.D. (1997) Contribution of partner switching and SpoIIAA cycling to regulation of sigmaF activity in sporulating Bacillus subtilis. J. Bacteriol. 179: 3922-3927.

Malik, S.S., Luthra, A., and Ramachandran, R. (2009) Interactions of the M. tuberculosis UsfX with the cognate sigma factor SigF and the anti-anti sigma factor RsfA. Biochim. Biophys. Acta 1794: 541-553.

Mao, X.-M., Ren, N.-N., Sun, N., Wang, F., Zhou, R.-C., Tang, Y., and Li, Y.-Q. (2014) Proteasome involvement in a complex cascade mediating SigT degradation during differentiation of Streptomyces coelicolor. FEBS Lett. 588: 608-613.

Mao, X.-M., Sun, N., Wang, F., Luo, S., Zhou, Z., Feng, W.-H., et al. (2013) Dual positive feedback regulation of protein degradation of an extra-cytoplasmic function $\sigma$ factor for cell differentiation in Streptomyces coelicolor. J. Biol. Chem. 288: 31217-31228.

Marles-Wright, J., Grant, T., Delumeau, O., van Duinen, G., Firbank, S.J., Lewis, P.J., et al. (2008) Molecular architecture of the "stressosome," a signal integration and transduction hub. Science 322: 92-96.

Marles-Wright, J. and Lewis, R.J. (2010) The stressosome: molecular architecture of a signalling hub. Biochem. Soc. Trans. 38: 928-933.

Martínez, L.F., Bishop, A., Parkes, L., Del Sol, R., Salerno, P., Sevcikova, B., et al. (2009) Osmoregulation in Streptomyces coelicolor: modulation of SigB activity by OsaC. Mol. Microbiol. 71: 1250-1262.

Martínez-Salazar, J.M., Salazar, E., Encarnación, S., Ramírez-Romero, M.A., and Rivera, J. (2009) Role of the extracytoplasmic function sigma factor RpoE4 in oxidative and osmotic stress responses in Rhizobium etli.J. Bacteriol. 191: 4122-4132.

Masuda, S., Murakami, K.S., Wang, S., Anders Olson, C., Donigian, J., Leon, F., et al. (2004) Crystal Structures of the ADP and ATP Bound Forms of the Bacillus Anti- $\sigma$ Factor SpoIIAB in Complex with the Anti-anti- $\sigma$ SpoIIAA. J. Mol. Biol. 340: 941-956.

Mattoo, S., Yuk, M.H., Huang, L.L., and Miller, J.F. (2004) Regulation of type III secretion in Bordetella. Mol. Microbiol. 52: 1201-1214.

Mercer, R.G. and Lang, A.S. (2014) Identification of a predicted partner-switching system that affects production of the gene transfer agent RcGTA and stationary phase viability in Rhodobacter capsulatus. BMC Microbiol. 14: 71.

Mitchell, J.E., Oshima, T., Piper, S.E., Webster, C.L., Westblade, L.F., Karimova, G., et al. (2007) The Escherichia coli Regulator of Sigma 70 Protein, Rsd, Can Up-Regulate Some StressDependent Promoters by Sequestering Sigma 70. J. Bacteriol. 189: 3489-3495.

Mittenhuber, G. (2002) A phylogenomic study of the general stress response sigma factor sigmaB of Bacillus subtilis and its regulatory proteins. J. Mol. Microbiol. Biotechnol. 4: 427-452.

Morris, A.R. and Visick, K.L. (2010) Control of biofilm formation and colonization in Vibrio fischeri: a role for partner switching? Environ. Microbiol. 12: 2051-2059.

Morris, A.R. and Visick, K.L. (2013a) Inhibition of SypG-Induced Biofilms and Host Colonization by the Negative Regulator SypE in Vibrio fischeri. PLOS ONE 8:.

Morris, A.R. and Visick, K.L. (2013b) The response regulator SypE controls biofilm formation and colonization through phosphorylation of the syp-encoded regulator SypA in Vibrio fischeri. Mol. Microbiol. 87: 509-525. 
NCBI Resource Coordinators (2016) Database resources of the National Center for Biotechnology Information. Nucleic Acids Res. 44: D7-19.

Norsworthy, A.N. and Visick, K.L. (2015) Signaling between two interacting sensor kinases promotes biofilms and colonization by a bacterial symbiont. Mol. Microbiol. 96: 233-248.

Österberg, S., del Peso-Santos, T., and Shingler, V. (2011) Regulation of alternative sigma factor use. Annu. Rev. Microbiol. 65: 37-55.

Paget, M.S. (2015) Bacterial Sigma Factors and Anti-Sigma Factors: Structure, Function and Distribution. Biomolecules 5: 1245-1265.

Paget, M.S.B. and Helmann, J.D. (2003) The sigma70 family of sigma factors. Genome Biol. 4: 203.

Pané-Farré, J., Lewis, R.J., and Stülke, J. (2005) The RsbRST stress module in bacteria: a signalling system that may interact with different output modules. J. Mol. Microbiol. Biotechnol. 9: 65-76.

Parida, B.K., Douglas, T., Nino, C., and Dhandayuthapani, S. (2005) Interactions of anti-sigma factor antagonists of Mycobacterium tuberculosis in the yeast two-hybrid system. Tuberc. Edinb. Scotl. 85: 347-355.

Park, Y.-H., Lee, C.-R., Choe, M., and Seok, Y.-J. (2013) HPr antagonizes the anti- $\sigma 70$ activity of Rsd in Escherichia coli. Proc. Natl. Acad. Sci. U. S. A. 110: 21142-21147.

Park, Y.H., Um, S.H., Song, S., Seok, Y.J., and Ha, N.C. (2015) Structural basis for the sequestration of the anti- $\sigma(70)$ factor Rsd from $\sigma(70)$ by the histidine-containing phosphocarrier protein HPr. Acta Crystallogr. D Biol. Crystallogr. 71: 1998-2008.

Pereira, S.F.F., Goss, L., and Dworkin, J. (2011) Eukaryote-like serine/threonine kinases and phosphatases in bacteria. Microbiol. Mol. Biol. Rev. MMBR 75: 192-212.

Price, C.W. (2011) The general stress response in Bacillus subtilis and related Gram-positive bacteria. In, Bacterial Stress Responses, 2 nd edition, ASM. Hengge Regine, Storz Gisela, p. 301.

Romero, P.R. and Karp, P.D. (2004) Using functional and organizational information to improve genome-wide computational prediction of transcription units on pathway-genome databases. Bioinforma. Oxf. Engl. 20: 709-717.

Sachdeva, P., Narayan, A., Misra, R., Brahmachari, V., and Singh, Y. (2008) Loss of kinase activity in Mycobacterium tuberculosis multidomain protein Rv1364c. FEBS J. 275: 6295-6308.

Sauviac, L., Philippe, H., Phok, K., and Bruand, C. (2007) An extracytoplasmic function sigma factor acts as a general stress response regulator in Sinorhizobium meliloti. J. Bacteriol. 189: 4204-4216.

van Schaik, W., Tempelaars, M.H., Zwietering, M.H., de Vos, W.M., and Abee, T. (2005) Analysis of the Role of RsbV, RsbW, and RsbY in Regulating $\sigma \mathrm{B}$ Activity in Bacillus cereus. J. Bacteriol. 187: 5846-5851.

Sharma, A.K., Rigby, A.C., and Alper, S.L. (2011) STAS domain structure and function. Cell. Physiol. Biochem. 28: 407-422.

Shi, L., Bischoff, K.M., and Kennelly, P.J. (1999) The icfG Gene Cluster of Synechocystis sp. Strain PCC 6803 Encodes an Rsb/Spo-Like Protein Kinase, Protein Phosphatase, and Two Phosphoproteins. J. Bacteriol. 181: 4761-4767.

Shi, Y. (2009) Serine/threonine phosphatases: mechanism through structure. Cell 139: 468-484.

Sineva, E., Savkina, M., and Ades, S.E. (2017) Themes and variations in gene regulation by extracytoplasmic function (ECF) sigma factors. Curr. Opin. Microbiol. 36: 128-137.

Smith, T.G. and Hoover, T.R. (2009) Deciphering bacterial flagellar gene regulatory networks in the genomic era. Adv. Appl. Microbiol. 67: 257-295. 
Sorenson, M.K., Ray, S.S., and Darst, S.A. (2004) Crystal structure of the flagellar sigma/antisigma complex sigma(28)/FlgM reveals an intact sigma factor in an inactive conformation. Mol. Cell 14: 127-138.

Staroń, A., Sofia, H.J., Dietrich, S., Ulrich, L.E., Liesegang, H., and Mascher, T. (2009) The third pillar of bacterial signal transduction: classification of the extracytoplasmic function (ECF) sigma factor protein family. Mol. Microbiol. 74: 557-581.

Thompson, C.C., Griffiths, C., Nicod, S.S., Lowden, N.M., Wigneshweraraj, S., Fisher, D.J., and McClure, M.O. (2015) The Rsb Phosphoregulatory Network Controls Availability of the Primary Sigma Factor in Chlamydia trachomatis and Influences the Kinetics of Growth and Development. PLoS Pathog 11: e1005125.

Valentini, M., Laventie, B.-J., Moscoso, J., Jenal, U., and Filloux, A. (2016) The Diguanylate Cyclase HsbD Intersects with the HptB Regulatory Cascade to Control Pseudomonas aeruginosa Biofilm and Motility. PLoS Genet. 12: e1006354.

Voelker, U., Voelker, A., and Haldenwang, W.G. (1996) Reactivation of the Bacillus subtilis antisigma B antagonist, RsbV, by stress- or starvation-induced phosphatase activities. J. Bacteriol. 178: 5456-5463.

Wuichet, K. and Zhulin, I.B. (2010) Origins and Diversification of a Complex Signal Transduction System in Prokaryotes. Sci. Signal. 3: ra50.

Yuan, A.H., Gregory, B.D., Sharp, J.S., McCleary, K.D., Dove, S.L., and Hochschild, A. (2008) Rsd family proteins make simultaneous interactions with regions 2 and 4 of the primary sigma factor. Mol. Microbiol. 70: 1136-1151.

\section{Figures legends}

\section{Figure 1: Domain organization of PSS modules of chosen bacterial species.}

The color code is: red for $\sigma$ factors, purple for anti- $\sigma$ factor domains, green for anti- $\sigma$ factor antagonists (anti-anti- $\sigma$ factors) and yellow for phosphatase domains. HAMP domains (present

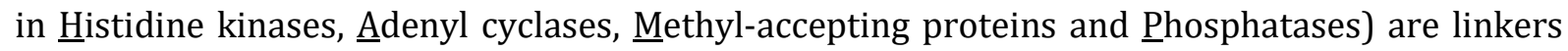
possessing a role in signal transduction. Transmembrane domains of membranous proteins are mentioned by "TM" (in black), other proteins are cytoplasmic. REC stands for Receiver domain.

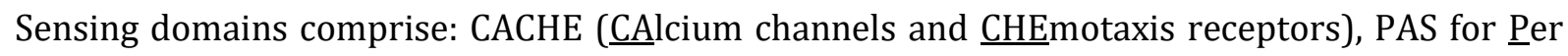
(Period Circadian Protein), A Arnt (Aryl hydrocarbon Receptor Nuclear Translocator protein), $\underline{\text { Sim }}$

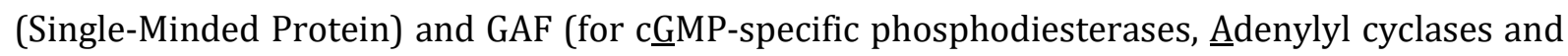
FhlA). When known, the physiological role of PSS is indicated as well as its sensing modules. The domain organizations appear on the right part of the figure. 

transduction systems.

The signal transduction pathways are divided in three major steps:

1(blue) - Signal sensing. Modules involved in the signal detection present a large range of mechanisms with various level of complexity. This step is the most diversified and bacteria have been very creative to detect signals and transduce them to the PSS modules.

2 (green) - Intermediate signal transduction from the input (signal sensing) to the output ( $\sigma$ factors). The partner-switching mechanism is highly conserved. The rule is that according to the environmental conditions, the $\sigma$ factor should be sequestered or released to hamper or allow its regulatory activity, respectively.

3 (red) - The $\sigma$ factor involved in the GSR. The release of the $\sigma$ factor leads to the expression of the genes belonging to the $\sigma$ factor regulon.

Question marks (?) indicate that the steps have not been experimentally demonstrated. means that the components are not conserved in all alpha-proteobacterial species. A green arrow represents a phosphorylation and a red arrow a dephosphorylation event. "HK-CA" corresponds to the domains HisKA and HATPase involved in receiver (REC) phosphorylation. Histidine kinases from two-component systems and the CheA1 kinase from the Che1 chemosensory system are represented. Protein names are indicated inside the drawing except if the protein harbors multiple domains, in this case the name is written above. The cytoplasmic membrane is symbolized in dark. The colors of the $\sigma$ factors and the PSS components are those of figure 1. Protein hampering is indicated by a line ending by a small horizontal line. $\alpha \sigma$ and $\alpha \alpha \sigma$ stand for anti-sigma factor and anti-anti-sigma factor (or anti-sigma factor antagonist).

\section{Figure 3: Occurrence and synteny of CrsR homologs in bacteria}

Searches for homologous proteins to S. oneidensis CrsR were performed using the bioinformatics BLAST tool from the NCBI database (NCBI Resource Coordinators, 2016) and the sequences were assembled using the program "Phylogeny" (Dereeper et al., 2008). Among the Gammaproteobacteria, CrsR homologs are found in Alteromonadales, Chromatiales, Methylococcales, 
725 Oceanospirillales, Pseudomonadales, Thiotrichales, Vibrionales. Symbol “*” indicate a genus. 726 Among the genus Pseudomonas, the species $P$. aeruginosa, P. putida, P. chlororaphis, $P$. 727 fluorescens, $P$. syringae, $P$. stutzeri were selected. The symbol “**” indicates that the synteny is conserved in all Pseudomonas species except for P. aeruginosa. The genus Vibrio includes $V$. mimicus, V. cholerae, V. vulnificus, and the genus Shewanella includes S. xiamenensis, $S$. decolorationis, S. sp. HN-41, S. baltica 0S185, S. sp. ANA-3, S. sp. MR-7, S. putrefaciens and S. oneidensis MR-1. Genes surrounding crsR homologs were examined by hand using the BioCyc database (Romero and Karp, 2004; Krummenacker et al., 2005). The question mark (?) means that the genes surrounding $c r s R$ homologs are not exhaustive because of the incomplete database. "HK" stands for Histidine Kinase, "RR" for Response Regulator and "RR-GGDEF" for a receiver domain fused to a GGDEF domain. GGDEF domains have an enzymatic activity producing c-di-GMP necessary for biofilm formation. The corresponding proteins and the color code of the bacterial species are summarized in the bottom of the figure. Inside gene drawings, 3 and 5 indicate the number of copies. 
Figure 1

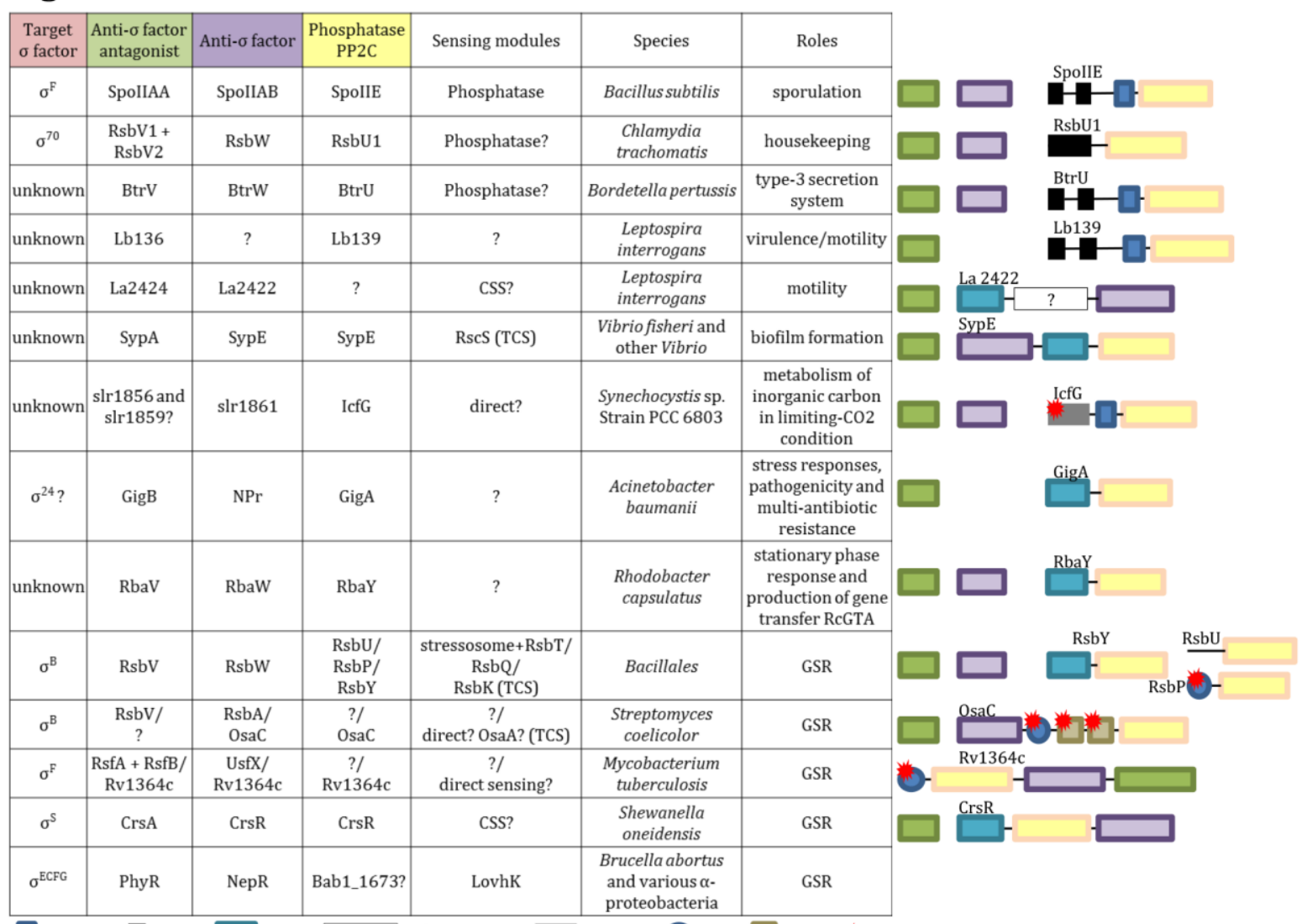


Figure 2
A Bacillus subtilis
B Bacillus cereus
C Streptomyces coelicolor
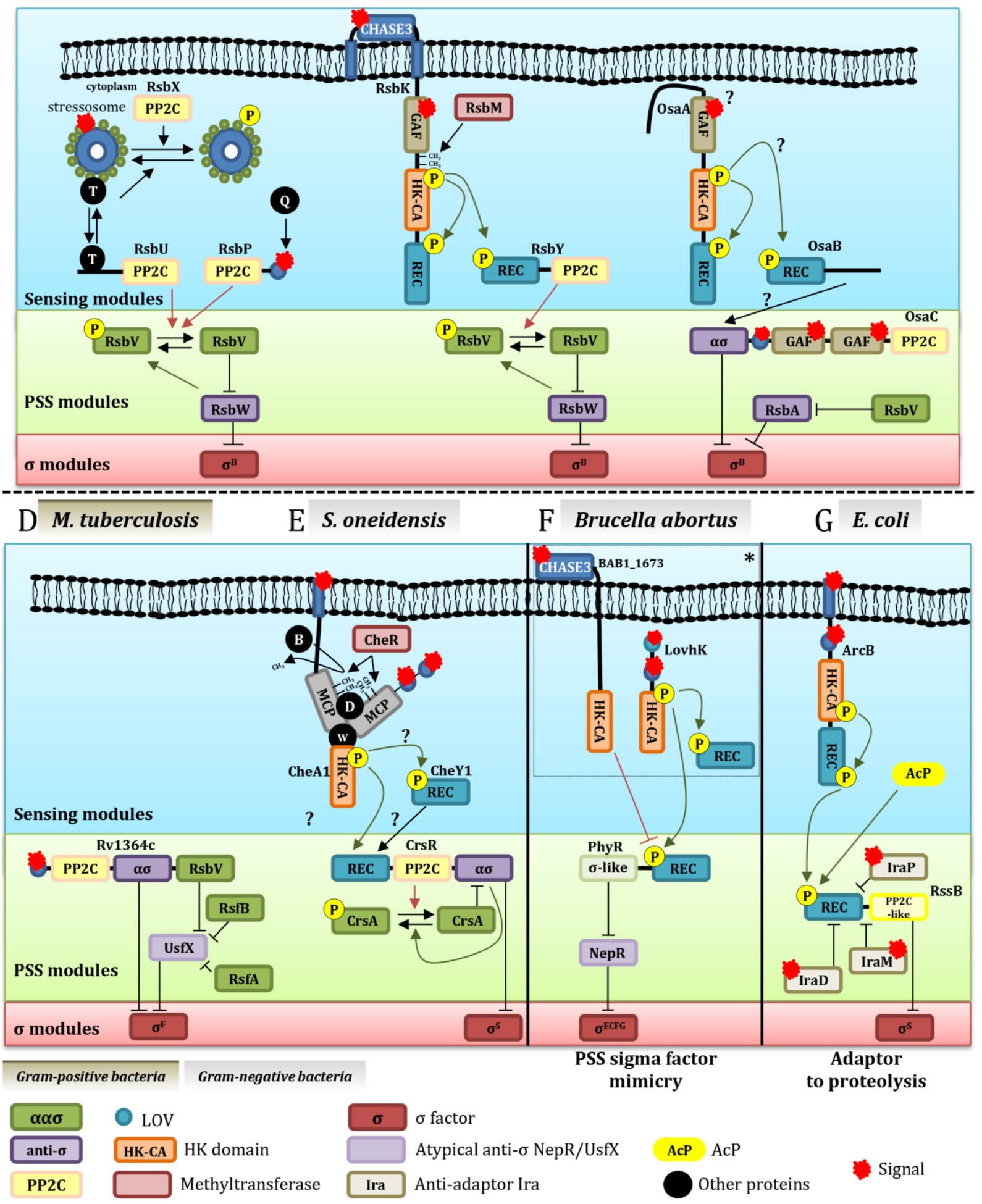


\section{Figure 3}

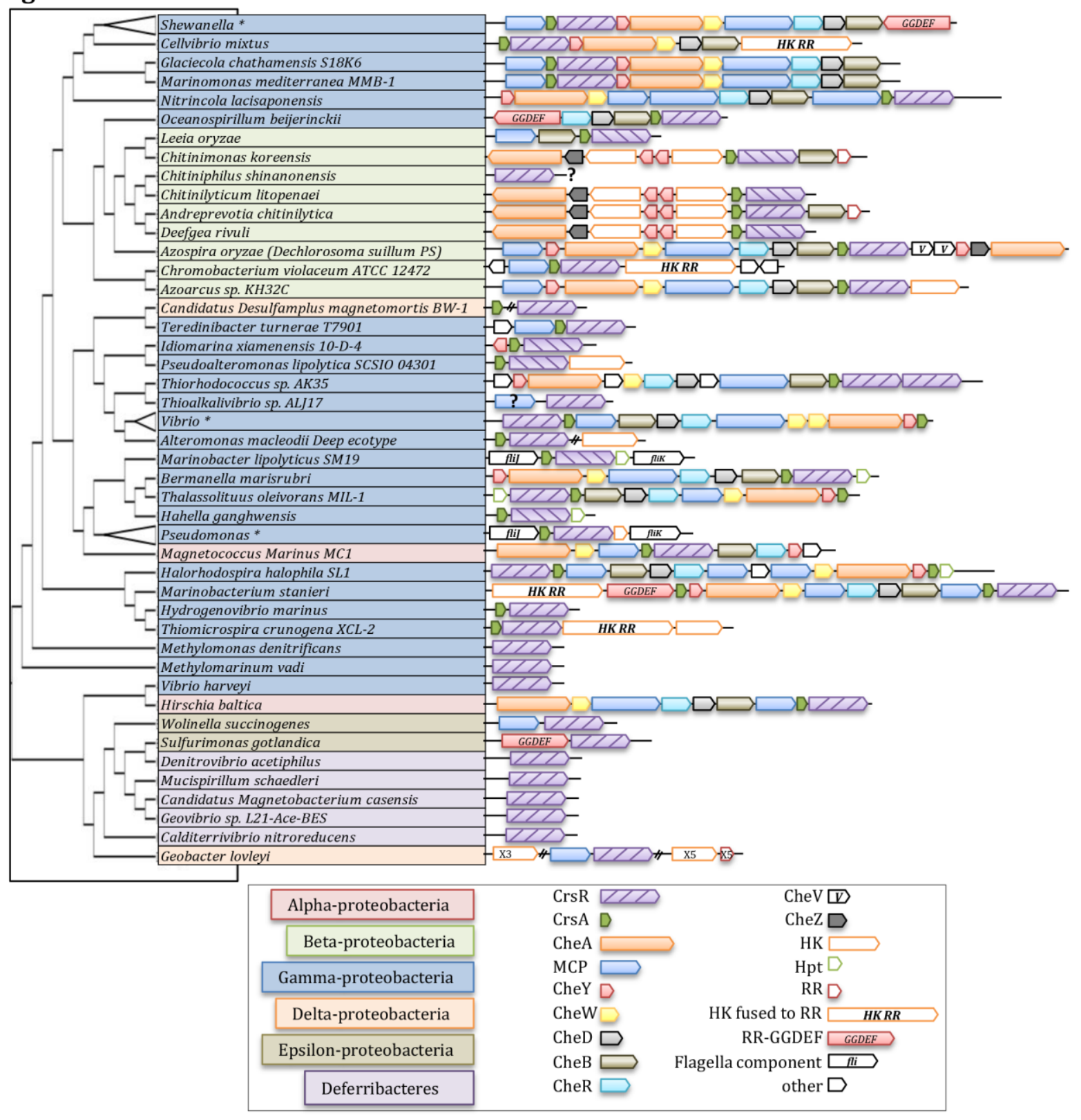

